This is a self-archived - parallel published version of this article in the publication archive of the University of Vaasa. It might differ from the original.

\title{
Monitoring and Protection of Power Distribution Networks with IEC 61850-9-2 Standard
}

Author(s): Palizban, Omid; Mekkanen, Mike; Kauhaniemi, Kimmo

Title: $\quad$ Monitoring and Protection of Power Distribution Networks with IEC 61850-9-2 Standard

Year: $\quad 2020$

Version: Accepted manuscript

Copyright (C)2020 IET. This paper is a postprint of a paper submitted to and accepted for publication in 15 th International Conference on Developments in Power System Protection (DPSP 2020) and is subject to Institution of Engineering and Technology Copyright. The copy of record is available at the IET Digital Library.

Please cite the original version:

Palizban, O., Mekkanen, M. \& Kauhaniemi, K. (2020). Monitoring and Protection of Power Distribution Networks with IEC 61850-9-2 Standard. 15th International Conference on Developments in Power System Protection (DPSP 2020), 2020, 1-6. https://doi.org/10.1049/cp.2020.0073 


\title{
Monitoring and Protection of Power Distribution Networks with IEC 61850-9-2 Standard
}

\author{
Omid Palizban $^{1^{*}}$,Mike Mekkanen ${ }^{1 *}$,Kimmo Kauhaniemi ${ }^{1}$ \\ ${ }^{1}$ University of Vaasa, School of Technology and Innovations, Vaasa, Finland
}

Keywords: Data processing, Modern grid, IEC 61850 standard, Power system protection, Sampled Values,

\begin{abstract}
In the modern power grid, communication plays a vital role in making the system more reliable and secure. The communications network transfers data between different units to a control center or to Intelligent Electric Devices (IEDs) in order to accurately control or protect the modern power system. The paper discussed possible ways to monitor the measured values for protecting the power system according to IEC 61850-9-2 (sample value). The sequence of the process consists of streaming the sample value packet, decoding it, and phasor extraction and filtering, which are discussed here. To validate the research, Sundom Smart Grid was used as a pilot to measure the values converted to digital form (IEC 61850-9-2) and to stream it to the system through a highly secure communications network. To decode the SV packet, two scenarios are presented in the case study, based on processing useful features of the IEC 61850-9-2 standard-based raw data streamed from the SSG, including $\mu$ PMU in a range of embedded instruments and a real-time simulator. The main result of this paper is an algorithm for analyzing SV packets and determining whether the network is operating normally.
\end{abstract}

\section{Introduction}

Modern protection relays operate on different principles than their classic counterparts, with differences in the processing of data received from instruments, such as Current and Voltage Transformers (CT and VT). The basic principle involves converting the received measurements as magnitudes and phasors and then compare them against the set values (thresholds). A start or trip signal is then sent if there is any deviation. In conventional protection relays, measured values are analog and are converted to digital and a series of samples prior to further processing. However, in protection and control of modern grids, the measured values of voltage and current are converted to digital format at the measuring point and streamed through a highly secure communications network. A basic block diagram of a digital protection relay is shown in Fig.1. Indeed, after feeding the functional measurement to the IEDs, the next step - the crucial step in protection - is data processing, which needs a measurement algorithm. The mathematical algorithm is implemented in the microprocessor of the IED. Neither very frequent nor very infrequent samples are suitable for protection relays, so the main question here is related to the sample rate of the signal. [1]

An on going project at the University of Vaasa, entitled FUture Smart Energy (FUSE), has brought together eight universities, research institutes, and companies from Finland and Germany to collaborate on developing technologies in the modern grid [2]. The project explores and demonstrates the application of technologies based on Artificial Intelligence (AI) to increase the resilience and general stability of the future energy grid, which will have to cope with an increasing percentage of renewable energy sources. A part of the project involves research and development of the method to receive early warning of developing faults, (ie. condition monitoring). The methodology can enable faster and more secure protection, even proactive protection for the modern grid. The main research questions relate to the data required from measuring points, a methodology for transferring these data from the measuring point to the IEDs, and technologies for processing the collected data at the IEDs. For protection, the two functional measurements are current and voltage, measured by current and voltage transformers respectively. In this project, the measured data is then converted to a digital format at the measuring point using a Merging Unit (MU) based on the IEC 61850-9-2 (sampled values) standard.

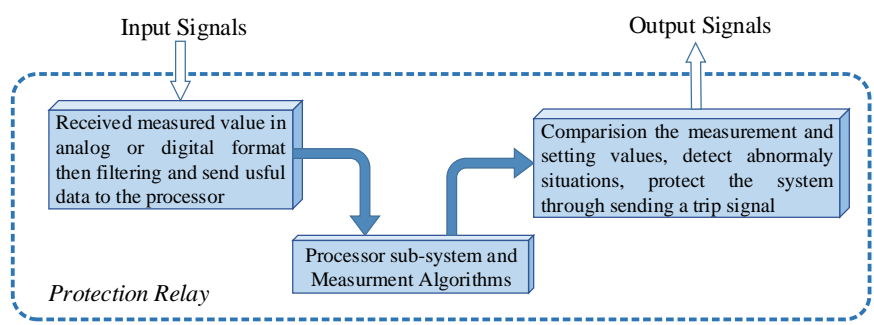

Fig.1 Block diagram of the protection relay

The IEC61850 standard, entitled "Communications networks and Systems for Power Utility Automation", is used for communications networks because it allows easy configuration and maintenance of the digital substation. One of the major features of the IEC 61850 communications network is the implementation of a publish-subscribe model 
of data transfer. Any device supporting IEC 61850 can then connect to the network and interface with other devices. When a device or application is added to the network, a message is published to the system and can be retrieved by subscribers anywhere on the network [3]. The main objective of the paper is to present possible methodologies for capturing data at the IED, along with data processing techniques for analyzing the data. In this regard, an algorithm is presented here to describe the process and it is also validated with real data.

The paper is organized as follows: the hierarchical architecture of data processing used in FUSE is presented in Section 2. The smart grid pilot is described in Section 3. The smart grid system converts the measured values from the measurement instrument to sampled values and streams them through the communications network. Section 4 discusses the data processing algorithm for protection relays; this involves the information available in an SV packet, methods of capturing values in a smart grid laboratory, and processing and analyzing the data. Finally, the paper is concluded in Section 5.

\section{Hierarchical Architecture of Data Processing}

The hierarchical architecture of processing nodes with advanced, AI-based data processing and reasoning capabilities consists of three levels. On the first level (level I), raw system performance data are collected by the "digital twins" from assets, and the data is processed for the use of the second level (level II) where the artificial intelligence tools are used to judge the system state. Finally, on level III, data from multiple Level II nodes are brought together in a control system to make them available for the operator. These three level are shown in Fig. 2 and are described on following subsections.

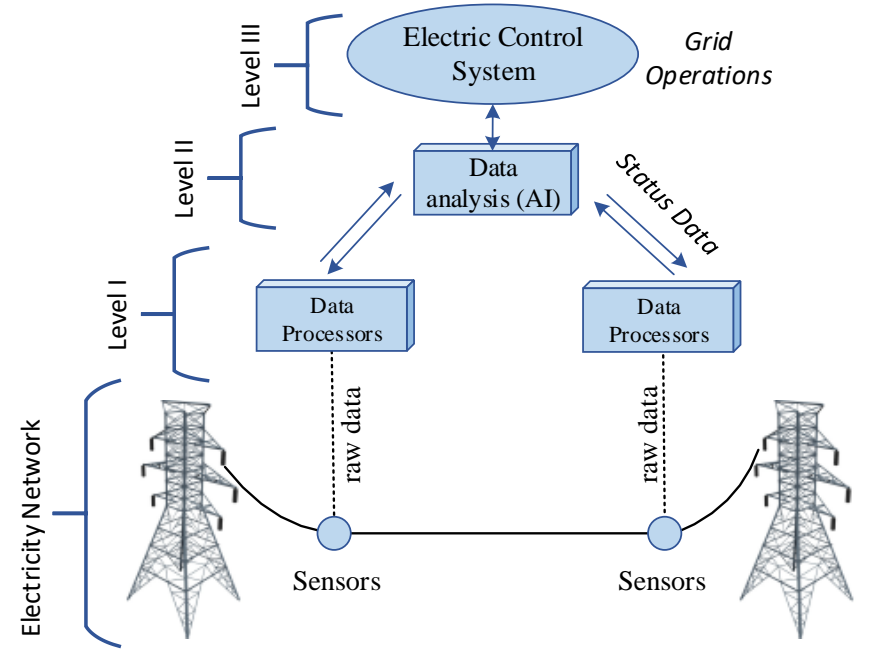

Fig .2 Concept of the hierarchical FUSE infrastructure for MV grids

\subsection{First level (level I)}

The main implementation target on this level is the collection of data from different parts of a system, such as power lines, transformers, and feeders, by the digital twins, which are a simplified model of the assets. The collection is implemented by smart sensors and the data is separated by smart algorithms in the digital twins. On this level, the system monitor detects deviations from set values. If an error is identified in the system, information on the deviation is transferred to level II through a communication network.

\subsection{Second level (level II)}

On level II, the data received from several digital twins is extracted and processed. Recognizing faults is the main goal of the level and it is achieved by data processing. The data processing can also identify errors and faults before they have an effect on the system, and also can help to schedule proactive maintenance for the system. The data collected from the sensors is fused with all the other information received from the parts associated with grid management decisions and maintenance. All the additional information can be used to determine whether the system is operating under normal or abnormal conditions. The level's outputs are sent to levels I and III to improve the performance of the system (the trip signal can also serve as feedback) or to make a decision, respectively.

\subsection{Third level (level III)}

The third level is the decision-making level of the architecture for data processing. The data processed on the second level is sent here to visualize the reasoning systems and to make a decision. Decisions can be made automatically by the electrical control system or manually by a human. After the information is received and compared with the standard and set points, it possible to make a decision that will optimize the system's operation. For example, if a feeder shuts down through a protection relay due to a fault in the feeder in the MV side, a soft control signal can be sent to the LV side to reduce consumption by load shedding on the customer side.

\section{Project implementation}

To utilize a practical data in the research, a smart grid pilot was employed. Sundom Smart Grid (SSG) is located in Vaasa, Finland, and has been developed through collaboration between ABB Oy, a local DSO (Vaasan Sähkö), a telecommunication company (Elisa), and the University of Vaasa. The main target of the smart grid pilot is more accurate fault detection in a distributed grid with modern grid automation, which can lead to increased system reliability. The SSG has been supported mostly by renewable energy sources. The Sundom Smart Grid pilot includes one primary HV/MV substation, four secondary $\mathrm{MV} / \mathrm{LV}$ substations, and two distributed generation (DG) units connected to the SSG: full power converter-based wind turbine $(3.6 \mathrm{MW})$ in the $\mathrm{MV}$ network and an inverter-based PV unit $(33 \mathrm{~kW})$ in the LV network. The currents and voltages are measured at the primary substations and at each secondary substation and transferred using the IEC 61850 9-2 protocol to the premises of project partners for research purposes. To protect and operate the SSG, several IEDs are used in substations, capturing the data from the instruments. A line diagram of the SSG is shown in Figure 3. [4] 


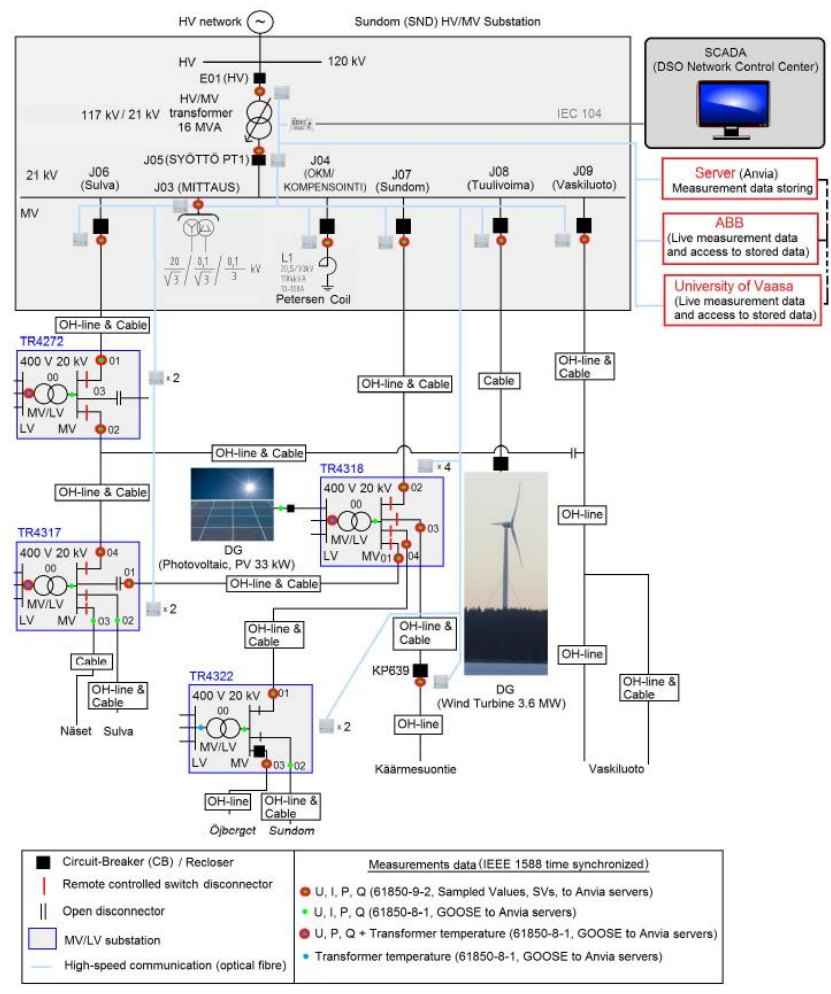

Fig.3 A line diagram of the SSG

\section{Data Processing Methodology}

As mentioned in Section 1, the main result of this paper is an algorithm for analyzing the received data (SV packets) and to determine whether the network is operating normally. The performance of the measurement algorithm is a major factor in successfully protecting the network. The algorithms utilized in this project are shown in Figure 4. Firstly, the SV packetwhich contains a value measured by instrument transformers or sensors - is received on level I node and decoded. Most of the protection functions implement the algorithm on the basis of the phasor quantity. In this project, a Discrete Fourier Transform (DFT) is used to extract the phasor values to estimate the required characteristics of the input signals. The phasor value is then used to determine the potential of an abnormality in the system. The phasor extraction is made using a sliding window of all the sampled values from the fundamental cycle $(20 \mathrm{~ms})$. If no abnormality is detected the next SV packet is processed and added to the window while discarding the oldest sample. In case of detection of abnormal values, the characteristics of the measured quantity are examined to detect and identify the fault. Different parts of the algorithm are described in following sections.

\subsection{Sample Value Packet}

The details of SV packets have been well described in [5] and [6]. Briefly, their structure and code can be divided into four parts: SV Ethernet frame, Application Protocol Data Unit (APDU), Application Subscriber Data Unit (ASDU), and a data set layer called PhsMeas1. To transmit SVs, a standardized Ethernet frame is used, holding APDU; the SV data is held by ASDU. An APDU can thus include several
ASDUs. To unify the data types and values in these two layers, the byte structures of APDU and ASDU are based on Abstract Syntax Notation One (ASN.1).

The SV data is thus encapsulated through coding and then streamed by the communication network. The rate of SV is either 80 or 256 samples/cycle. The SSG pilot uses 80 samples/cycle (4000 samples/second), giving a time interval for each SV packet of $250 \mu \mathrm{s}$ (1 s/4000 sample packets).

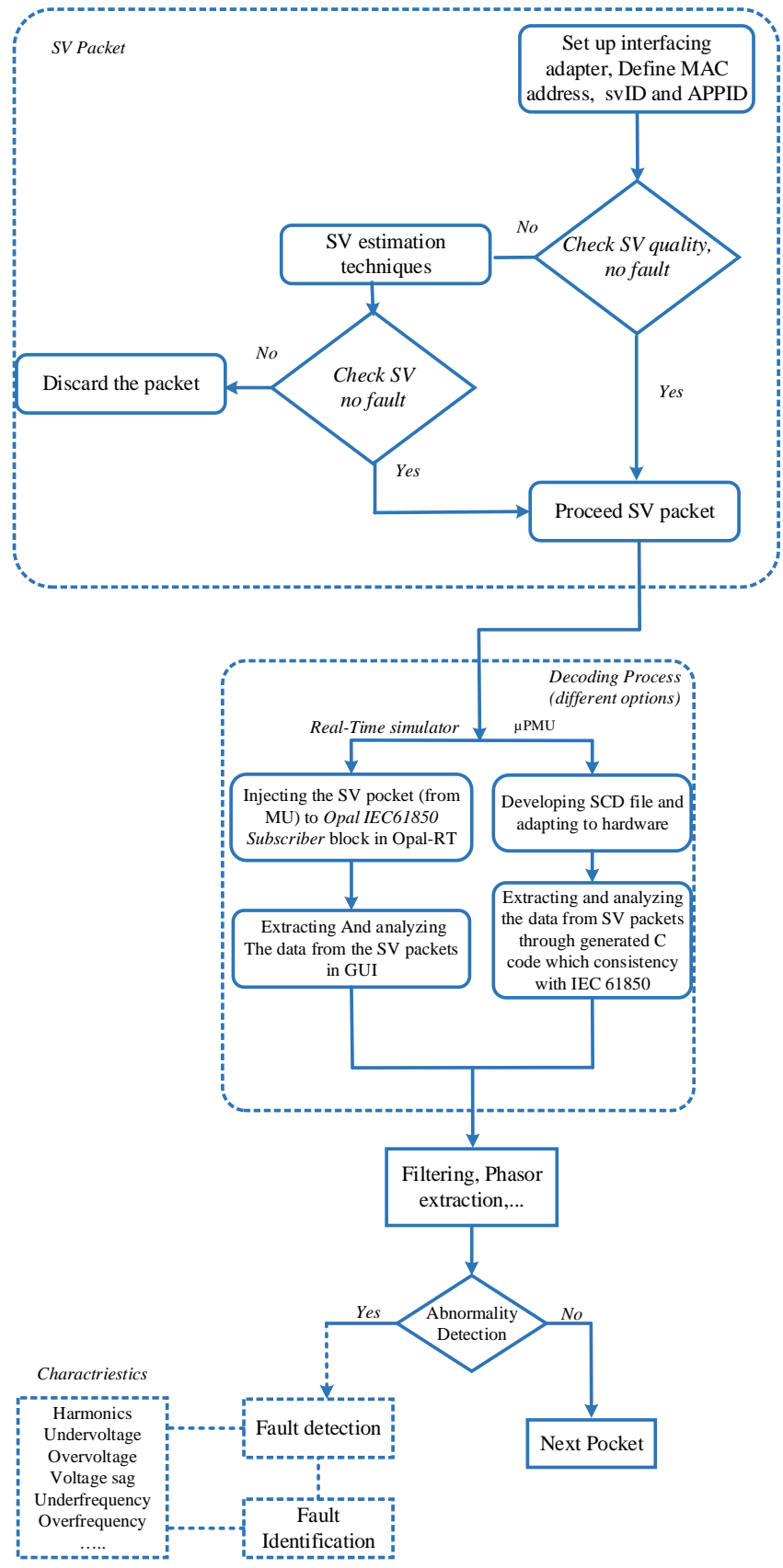

Fig.4 A flowchart of operations to transfer and capture data

The values measured at the secondary substation in the Sundom Smart Grid stream are in SV format in different packets and are captured by the smart grid laboratory at the University of Vaasa. The next step is to decode the SV packet so as to convert the value into raw data format. There are two 
scenarios for capturing the SV packet: a network capture tool and an SV block in a real-time simulator (OPAL-RT) in the laboratory. These two methods are described in detail in the following subsections.

\subsection{Capturing data at SSG}

One option for processing the useful features of the IEC 61850-9-2 standard-based raw data streamed from the SSG is to build up a real-time simulation model that consists of several merging unit (MU) blocks from the Opal-RT lab library (see Fig.5). This real-time simulation model has the ability to subscribe and analyze the received data (SV packets) to determine the status of the network of the suburban area Sundom Smart Grid. By bridging the existing physical entity (SSG) and the virtual real-time simulation model, data from SSG IEDs is transferred seamlessly, allowing the virtual entity to exist simultaneously with the physical entity. The features extracted from the SV packets can be used for different parallel monitoring, protection, and control applications when connectivity, interoperability, and data quality are sufficient. Integrating the ICT model with the SSG physical system model and creating a digital twin replica allows a low-risk solution in a lab, where multiple ICT fault scenarios can be evaluated and their impact on the operation of the power system can be assessed. Alternately, the digital twins can be directly connected with the field IEDs and control units to provide a robust testing, training, and research capability that can alleviate potential threats to critical physical infrastructure. A sequence of operations for monitoring raw data in the smart grid laboratory is shown in Figure 4 and described here:

a) Determining requirements for the simulation model, based on the case study;

b) Model development, which involves modelling the MU block-based SSG data sources, defining the APPID and svID for each source, extracting useful features from the received SV packets, encapsulating the extracted features, and transmitting them to other nodes over different IEC 61850 protocols (such as GOOSE or MMS);

c) Interfacing the model with real-time data over the Opal-RT Ethernet network adapters and running the model

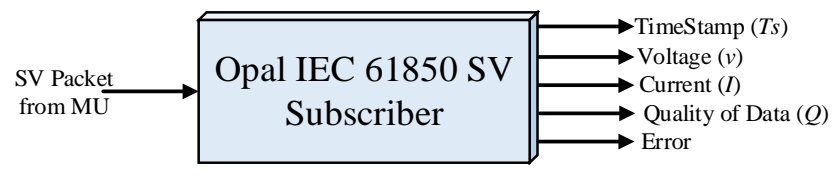

Fig.5 An Opal-RT block to extract the SV packets

The second option in the case study utilizes the useful features of the IEC 61850-9-2 standard-based raw data streamed from the SSG to develop $\mu$ PMU in a range of different embedded instruments. These embedded instruments subscribe to the IEC 61850-9-2 SV stream from the SSG and extract the useful features, execute the control function (if any) inside the device, and share the data over other IEC 61850 communication protocols. Development of the $\mu \mathrm{PMU}$ had the following phases: a) The substation configuration description (SCD) file was developed and further adapted into hardware;

b) $\mathrm{A}$ C code representation of a model and communication instances tailored to this model was generated. In the generation process, each type of IED data model can be mapped directly to a $\mathrm{C}$ data structure, resulting in a hierarchy of data structures. The generated $\mathrm{C}$ files must also be accompanied by platform-specific code to ensure consistency with IEC 61850.

c) Several MUs based on the SSG SV stream subscriber were developed in $\mathrm{C}$ using open-source libraries, defining the parameters needed to be subscribed successfully

d) Extracting defined parameters (voltage and current) from the received SSG SV steam.

e) Running the extracting/analyzing algorithm in the hardware based on the achieved signal feature parameters. It then needs to be capsulated and shared to other nodes on different levels and over other IEC 61850 communication protocols.

\subsection{Phasor extraction, filtering}

The next step is phasor extraction and estimation, for which several methods - such as Discrete Fourier Transform (DFT), cosine and least squares algorithms, Kalman filtering, and wavelet transform - have been proposed over the years. DFT is used to determine phasors in the FUSE project; this approach is commonly used to estimate phasors in power system protection (relays), and uses Fourier analysis of shorttime variations [7]. DFT was thus applied to extract the phase angle and magnitude (peak value) of the fundamental frequency voltage and current. The method is employed for the data received during each fundamental cycle, so the number of SV packets for each analysis is 80 . The following equations perform DFT on an input SV packet, which is the fundamental frequency component from the measured values of voltage and current [8][9]:

$$
\begin{aligned}
& I(j \omega)=\frac{1}{N} \sum_{n=0}^{N-1} i_{n} e^{-j n \frac{2 \pi}{N}} \\
& V(j \omega)=\frac{1}{N} \sum_{n=0}^{N-1} v_{n} e^{-j n \frac{2 \pi}{N}}
\end{aligned}
$$

Where $i$ and $v$ are the instantaneous value of the current and voltage respectively. The number of samples in sliding window is $N$. The phasor of voltage and current are also presented with $I$ and $V$ respectively.

After extracting the phasor value and filtering, these components are used in the algorithm for analyzing the protection functions. The performance of the protection relay is very dependent on the mathematical algorithm used (and particularly the measurement algorithms), because the phasor values of the current and voltage (one or both) is used in the fault detection process in the protection relays. For instance, the current signal is used to detect faults in an overcurrent 
relay, whereas both the voltage and current signals are used in an impedance protection relay. The characteristics and parameters needed for a range of applications (based on the IEC 61000 standard) should be considered when implementing the monitoring and protection functionality in the system. Moreover, some optional approaches could help to increase the performance of the protection. For instance, active and reactive power, frequency, and the harmonics for current and voltage are used to investigate power quality applications in the power system, which makes it possible to detect faults before they happen and to improve fault location by monitoring parameters (such as variations) from different feeders.

\subsection{Real-Time Monitoring}

Using real-time monitoring, particularly $\mu \mathrm{PMU}$, as the merging unit SV subscriber makes it possible to optimize the use of different types of microcontroller and coding languages, such as a Raspberry Pi, Beagle Bone, Java, and C++. To ensure that the solution operates reliably, it will be necessary to develop and validate testing procedures. A simple lab setup of the test stand described here will allow the MU SV subscriber to be tested under different circumstances that reproduce a real merging unit's useful parameters when operating in a system; this is illustrated in Figure 6.

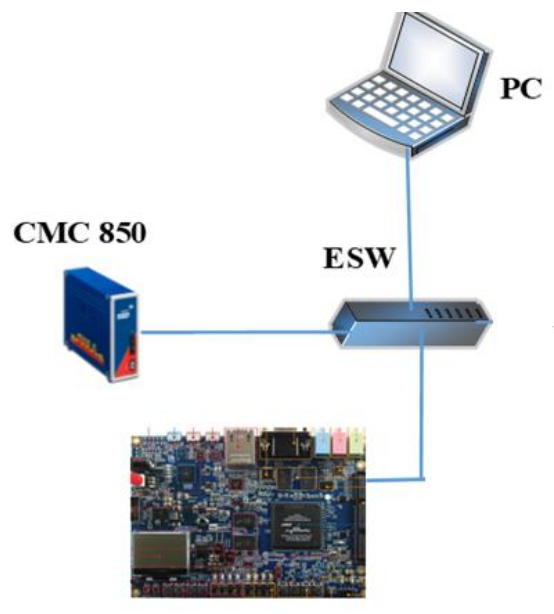

Fig.6 A setup for testing in the smart grid laboratory

The Altera SoC FPGA board operating as an SV subscriber is interfaced to the SV stream published by the CMC 850, and to a computer that controls the source of the SV and analyses the data extracted from the SV stream. The purpose of this set-up is to test and validate the soft MU SV subscriber implemented on the FPGA board, since the MU SV parameters are known (f, I and V) on the publishing side during the testing scenario, and can be compared to those on the subscribing side, i.e., the FPGA. Figures 7-8 use some of the extracted data $(80$ samples) to illustrate all the useful parameter that can be calculated, such as the instantaneous value of V and I, the RMS value, $\mathrm{f}$, the phase, and so on; these can then be used to predict whether the system is operating normally or not.

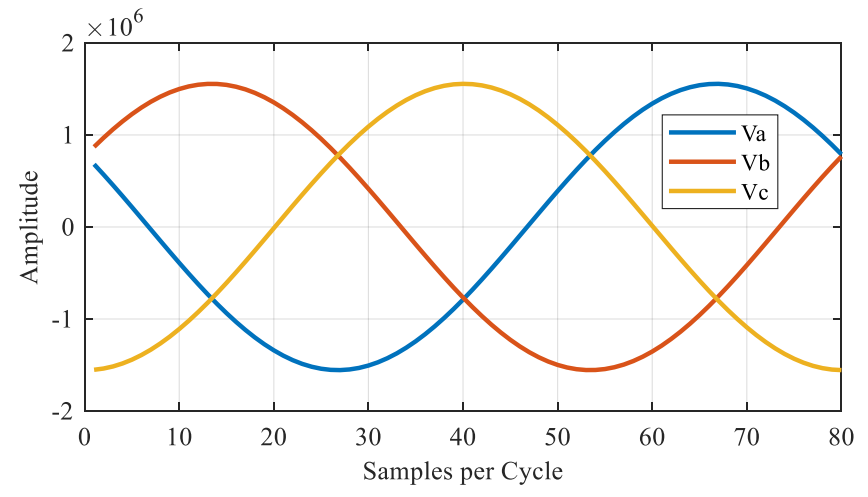

Fig.7 Subscribing to the voltage data in the smart grid laboratory

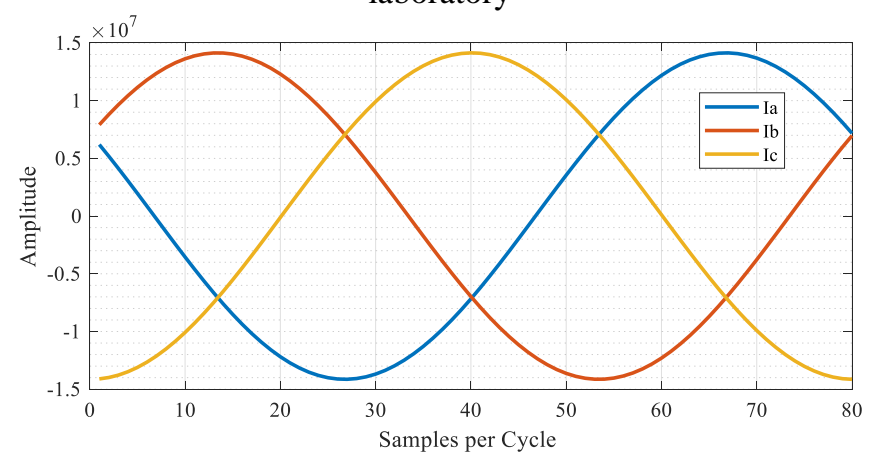

Fig. 8 Subscribing to the current data in the smart grid laboratory

\section{Conclusion}

New technologies are required to monitor, protect, and control modern power distribution systems. Measuring data for protection, employing data transfer techniques, and the methodologies for data processing are the major challenges in such a system. This paper has presented the sequence of the protection process in a modern power system and illustrated an algorithm to cover the entire process. The current and voltage values are measured by the instruments and converted to a sample value (IEC 61850-9-2), before being streamed through a communications network. Two methods for capturing the data are then explained, as well as methodologies for phasor extraction and filtering. The project is in an ongoing situation and a practical test for these proposed scenarios in under development. The results show that modern technology can protect the power system more accurately through the rapid transfer of data from measuring point to IEDs, and can be a part of network digitalization of substations. Investigating solutions for delayed or lost sampled value packets could be considered for future work. Moreover, it will also prove important to develop an algorithm for fault identification and fault detection.

\section{Acknowledgements}

The authors would like to acknowledge the financial support provided by the Finnish funding agency for research, the Business Finland, to the Future Smart Energy (FUSE) research program (Project number: 7038/31/2017). 


\section{References}

[1] M. N. Ibrahim, "Performance evaluation of measurement algorithms used in IEDs" University of Adelaide, 2012.

[2] "Future Smart Energy," https://www.fuse.ac/, 2019

[3] R. Hunt, B. Flynn, and T. Smith, "The Substation of the Future: Moving Toward a Digital Solution" IEEE Power Energy Mag., vol. 17, no. 4, pp. 47-55, Jul. 2019.

[4] P. Hovila, A. Monot, H. Laaksonen, and M. Ritakasari, "Advanced Utilization of Big Data for RealTime Monitoring and Data Analytics in Sundom Smart Grid" in 25th International Conference on Electricity Distribution, 2019, no. June, pp. 3-6.

[5] P. Zhao, "IEC 61850-9-2 Process Bus Communication Interface for Light Weight Merging Unit Testing Environment", M.Sc. Dissertation, KTH, Faculty of Technology, 2012.

[6] Damilola Simeon Adesina, "Interfacing IEC 61850 9-2 Process Bus Data to a Simulation Environment" Faculty of Technology, University of Vaasa, 2015.

[7] C. D. Prasad and P. K. Nayak, "A DFT-ED based approach for detection and classification of faults in electric power transmission networks" Ain Shams Eng. J., vol. 10, no. 1, pp. 171-178, 2019.

[8] S. N. Ananthan, A. F. Bastos, S. Santoso, and A. Del Rosso, "An automated technique for extracting phasors from protective relay's event reports" Energies, vol. 3, no. 4, pp. 1-18, 2018.

[9] M. S. Sachdev, F. Lopez, M. Meisinger, and J. Wang, " Underestanding Microprocessor-Based Technology Applied to Relaying”, Report of Working Group I-01 of the Relaying Practices Subcommittee, 2009. 